Brit. J. industr. Med., 1961, 18, 175.

\title{
A SURVEY OF THE METHODS DEVELOPED IN THE NATIONAL COAL BOARD'S PNEUMOCONIOSIS FIELD RESEARCH FOR CORRELATING ENVIRONMENTAL EXPOSURE WITH MEDICAL CONDITION
}

\author{
BY
}

J. W. J. FAY and J. R. ASHFORD

From the Pneumoconiosis Field Research Unit, National Coal Board, London

(RECEIVED FOR PUBLICATION APRIL 17, 1961)

The correlation of the medical and environmental data (i.e. the derivation of the dosage-response relationship) in a study such as the National Coal Board's Pneumoconiosis Field Research (P.F.R.) is subject to many complicating factors compared with the more conventional types of biological assay. Several methods have been developed within the Research to overcome these difficulties, and the new procedures are described. Each is concerned with the estimation of the direct relation between the radiological abnormality associated with simple pneumoconiosis and some single measure of the past hazard, but the basic techniques are sufficiently general to be applicable in other fields of study.

The first development involves the definition of an underlying continuous scale of radiological abnormality. This prepares the way for the derivation of the "quantitative" relation between exposure and response, to replace the "semi-quantal" relation which is inherent in the use of a small number of discrete categories of radiological abnormality. The effect of errors of observation of dosage and response on the corresponding quantitative and quantal relationships is then determined.

The second development concerns the use of a "multi-dimensional" representation of past hazard. Most of the men under observation had worked in a number of different mining occupations before their first chest radiograph was taken, but this exposure cannot be assessed in terms of dust concentrations, for which reliable data are not available. Nevertheless, it is shown that past hazard can usefully be represented by three "dimensions" corresponding to the periods spent in three main types of environment-(a) the coal-face (coal-getting shift), (b) the coal-face (preparation shift) and (c) elsewhere underground. Each man's past exposure up to the time of his first chest radiograph can be expressed in terms of these three dimensions and the effect of each environment separately can be determined.

The third development extends the multi-dimensional approach to cover not only the working history before the first medical examination, but also the recorded exposure (in terms of measured dust concentrations), to which each man has been subject between the first and subsequent "followup" surveys. This measured exposure is regarded as one dimension of the man's total exposure up to the time of his second (or later) examination, and it is possible in this way to determine the direct relation between radiological abnormality and measured exposure, even when this component represents only part of the total hazard to which the man has been subject.

The application of the methods is illustrated by the analysis of some of the data which have been obtained in the Pneumoconiosis Field Research.

One of the main objectives of the National Coal Board's Pneumoconiosis Field Research (P.F.R.) is to determine what environmental conditions should be maintained in British collieries if the miners are not to be disabled by the dust they breathe in the course of their work. The problem is 
being tackled by a study of the relation between the prevalence and progression of pneumoconiosis and the environmental conditions, particularly airborne dust concentrations, at a number of collieries located throughout the coalfields in the United Kingdom (Fay, 1957).

The population under examination comprises the underground and surface workers at 25 selected collieries, amounting to about 35,000 men in all. A series of radiological surveys is being made at each colliery at intervals of about four years. Since 1953, when the Research was started, a continuous programme of environmental measurement has been carried out at each colliery, thus providing a record of dust exposure to match against radiological change for each man (Fay and Rae, 1959).

The correlation of the resulting medical and environmental data presents a task of some magnitude and complexity. Dosage-response relationships in biological assay work are commonly expressed in terms of the probability of a single "yes - no" (quantal) response to a known stimulus, e.g. the death of a test animal as the result of the controlled administration of a given dose of a single poison, but this simple situation is not present in a study such as the Pneumoconiosis Field Research. For one thing, the data are "uncontrolled", in the sense that the observer cannot determine the "doses" applied, having instead to make the best use he can of whatever information he is able to derive from his study of the subjects under examination. The use of such "uncontrolled" data is, of course, by no means a new development; Bull and Squire (1949), for example, applied the method of probit analysis to the study of mortality in relation to area of burns. In the present investigation the position is further complicated by a number of important factors.

The subjects are under observation with respect to both radiological pneumoconiosis and dust exposure. The "response" is measured, not in terms of a simple "dead or alive" observation, but in terms of more than one discrete stage of severity, as represented by the various radiological categories of simple pneumoconiosis. An essentially continuous process, whereby increasing amounts of dust produce increasing amounts of abnormality, is thus restricted to expression in quantal terms, using the steps corresponding to the discrete categories of pneumoconiosis. Obviously it is desirable to remove this limitation, and to establish a quantitative relation between exposure and response. This involves the derivation of the underlying continuous scale of radiological abnormality, and this particular step is the first main development described in this paper. It is shown that by the choice of a suitable scale of abnormality a straight-line relation can be obtained between degree of pneumoconiosis (measured on a continuous scale) and a logarithmic function of environmental exposure. This quantitative description represents a generalization of the quantal relationships governing the separate responses in terms of category 1 or more or category 2 or more pneumoconiosis, and provides a better insight into the processes involved than the simpler quantal response technique based on the discrete category boundaries implicit in the I.L.O. scale (International Labour Organization, 1953). The application of the method is illustrated by means of data obtained on the initial medical survey at one of the Pneumoconiosis Field Research selected collieries for a group of men who had worked only in one particular occupation.

In studies such as this the estimates of radiological abnormality and past hazard used for the dosageresponse analysis may be subject to considerable errors of measurement. The effect of these errors on the quantitative abnormality-exposure relation is described and it is shown how the corresponding quantal relationship is modified by the uncertainties in the data.

The third major difficulty in the present study arises from the fact that the "dose" causing the response is not usually capable of expression in terms of a single parameter, because of the absence of any reliable quantitative information about exposure before the first medical surveys. It is, however, possible to obtain a measure of a man's past exposure from his working history, in terms of the periods spent in various types of environment. The majority of the men worked in more than one environment during the period before the initial medical surveys, and were thereby exposed to more than one level of hazard. It is therefore necessary to take account of a number of different "dimensions" of past exposure. A method of analysis has been developed to determine the dosage-response relationship associated with each dimension separately on the basis of the information from men with mixed past working histories. The application of this approach is illustrated by an analysis of the past exposure histories obtained from the whole population examined on the initial radiological survey at one of the Pneumoconiosis Field Research collieries. The advantages of this development will be immediately apparent, in that use is made of the data available for all subjects, instead of the analysis having to be restricted to the proportion (often small) of subjects who have been exposed to only one level of the hazard.

The information about past exposures up to the 
time of the first medical surveys is limited to the periods spent in different types of environment, as described above. Since then, however, an extensive programme of airborne dust sampling and measurement has been followed, and during the four years or so between the first and second radiological examinations each man's dust exposure has been continuously recorded in quantitative terms. Hence, a man's cumulative exposure since joining the coalmining industry can be regarded as a "multidimensional" variable, in terms of his different types of exposure before the first medical survey, together with one or more additional "dimensions" corresponding to his measured exposure between the first and second medical surveys. The use of the multidimensional method has therefore been extended to the analysis of the "progression" data, obtained from the follow-up medical surveys. In this way the probability that a man has developed a given category of simple pneumoconiosis or more at the time of (a) the first and (b) the second medical survey may be expressed as a function of the two sets of multi-dimensional variables, and the effect of the measured component alone may be assessed. The method has been developed to cover alternative procedures for reading the chest radiographs. Its use to estimate the relation between radiological abnormality and measured exposure is illustrated by means of the analysis of the data from the first and second radiological surveys at one of the Pneumoconiosis Field Research collieries. It must be emphasized that the figures quoted are used at this stage purely for the purposes of illustration of the method, and no importance should be attached in a quantitative sense to the results deduced.

In the following sections of this paper a general description is given of these various new methods, which are applicable in other fields than the study of coalworkers' pneumoconiosis. Full details of the underlying mathematical treatments have been published elsewhere (Ashford, 1958a; Ashford, 1959; Ashford, Smith, and Brown, 1960).

\section{Medical Data}

On each medical survey a 14 in. $\times 14$ in. posteroanterior (P.A.) chest radiograph is taken of every man examined, and the readings of these films are the source of information about the extent of the man's radiological pneumoconiosis. On the second and subsequent rounds of medical surveys supplementary measurements are also made of lung function, anthropometry, and respiratory symptoms (Rogan, Ashford, Chapman, Duffield, Fay, and Rae, 1961). The main application of the new methods of correlation is, however, in terms of the radiological readings, and the present paper is concerned only with this aspect, so far as the medical data are concerned.

The categories of pneumoconiosis which have been used in the medical surveys are those accepted by international agreement (International Labour Organization, 1953), which recognizes a distinction between simple and complicated pneumoconiosis [or progressive massive fibrosis (P.M.F.)]. Simple pneumoconiosis is subdivided on a quantitative basis into three categories, increasing in degree of abnormality and numbered 1 to 3, category 0 being reserved for films which are "within normal limits". Films showing evidence of complicated pneumoconiosis are classified separately into four categoriesA, B, C, and D. Because of the comparatively small number of men with P.M.F., and the uncertainties about the mechanism by which this condition becomes superimposed on simple pneumoconiosis (Gough, 1947; Cochrane, Cox, and Jarman, 1952), the methods of correlation described below are confined to the development of simple pneumoconiosis.

In view of the considerable element of doubt associated with a single reading of pneumoconiosis, even by experienced observers (Fletcher and Oldham, 1949), the final or "definitive" reading on each film is based on a series of readings by two doctors. The procedures applied and the results achieved have been described in detail elsewhere (Fay and Ashford, 1960; Ashford, 1960). Essentially, it has been the standard practice for each film to be read independently by the two doctors. Films on which these "routine" readings are not consistent are subsequently read again by both doctors in consultation and the definitive category is assigned as the result of their joint discussion. The readings of the films obtained on the second round of surveys have so far been made without reference to the corresponding first survey films, although consideration is currently being given to the possible application of other methods of reading. To check that the standards of reading on successive surveys at each colliery are comparable, a sample of the first survey films is read again at the time of the second survey. All the routine and definitive readings are made without reference to the man's past environmental history.

Although great care is exercised in taking and processing radiographs in order to maintain a consistent standard of radiographic technique, there are inevitably some variations in the quality of the films obtained on the various surveys. Superimposed upon these variations in producing the radiographs are the errors associated with the film reading process. 


\section{Environmental Data}

In view of the absence of sufficiently reliable environmental measurements at the selected collieries before the beginning of the Research, no quantitative information is available about the man's previous exposure to dust. However, at the time of the first medical survey a working history was obtained of each man presenting himself for examination, showing the time spent in each of his various occupations and working places since leaving school. This information was checked against an independently prepared history of the colliery and subsequently summarized by the use of an electronic computer (Hicks, Fay, Ashford, and Rae, 1961) in terms of the periods spent in each of the six main types of general environment [hard heading, coalface (coal-getting shift), coal-face (preparation shift), elsewhere underground, surface "dusty" and surface "non-dusty"] at the colliery in question and at other collieries. Each man is also assigned to a "pit" classification and "exposure" category on the basis of his past record, so that groups of men who have consistently followed the same occupation at the same colliery can be identified. These methods are, of course, an extension of the classical work done in South Wales by the Medical Research Council (Hart and Aslett, 1942). The working histories provide the only reliable information about the environmental exposures of the men before the first medical survey. This component will represent an appreciable part of the total exposure of the majority of the population for many years to come, and one of the main incentives for the development of new methods of correlation was the need to make the best possible use of these summarized past histories.

A systematic programme of environmental measurement has been carried out at each of the selected collieries since the Research started and the results obtained provide a basis for reliable estimates of individual exposures from the time of the initial medical surveys. The sampling procedure is based on an extension of the "random collier" method of Patterson (1939) and Oldham and Roach (1952). At each colliery the underground and surface populations are subdivided into a number of homogeneous strata, or occupational groups, consisting of men working at the same job, at the same place, at the same time. In this way the environment within each group may be expected to be reasonably uniform, and this is, of course, automatically checked by the subsequent measurements. By sampling a series of randomly selected individual workmen throughout a complete shift, the "exposure index" corresponding to each occupational group is determined, and this figure is attributed to each of the men belonging to the group, for the appropriate period which he spent in that group. Subsequent reference to the detailed record which is maintained of the attendance and movement from group to group of all men on the colliery register enables the individual environmental exposures to be calculated for any period of time after the first medical survey.

The dust concentrations are measured with the thermal precipitator and expressed in terms of the number of particles in the "respirable" size range per unit volume of air. The standard count is made in the 1-5 micron size range, and the relative proportions of "coal" and "non-coal" (i.e. mineral) are assessed. In addition, size distribution analyses are made on a proportion of the samples, in terms of the particle size ranges $0 \cdot 5-1 \mu, 1-2 \cdot 5 \mu, 2 \cdot 5-5 \mu$, and above $5 \mu$. These measurements are supplemented by compositional analyses of the respirable dust, using microscopic, $x$-ray diffraction, and chemical methods, and on this basis a considerable variety of alternative measures of exposure is available for investigation.

In summary, the total environmental exposure of each individual since joining the mining industry can be subdivided into two distinct components, representing respectively his working history up to the time of the first medical survey, expressed in terms of periods in different environments, and his subsequent exposure, based on measured dust concentrations and time at risk, expressed in terms of any one of a number of different parameters. When applied as a measure of environmental exposure the working histories may be regarded as subject to errors of measurement, due to both vagaries of memory and variations in the hazard corresponding to the same class of environment. The measured component of exposure is also subject to errors of estimation, which are controlled by systematic adjustment of the allocation of sampling shifts between the occupational groups (Ashford, 1958b).

\section{Relation between Prevalence of Simple} Pneumoconiosis and Environmental Exposure

The Underlying Relationship.-It is generally accepted that simple pneumoconiosis is caused by the inhalation and retention of airborne dust in the lungs. Although the exact way in which the process takes place is not clearly understood at the present time, it is reasonable to suppose that the characteristic radiological abnormalities will be related to the quantity and composition of the dust retained and/or any corresponding tissue reaction (Rivers, Wise, King, and Nagelschmidt, 1960). In coalworkers' pneumoconiosis the situation is complicated by the possible intervention of progressive massive fibrosis 
(P.M.F.), a condition which may be due to the superimposition of a tubercular infection upon a background of fairly advanced simple pneumoconiosis (Gough, 1947; Cochrane et al., 1952; Cochrane and Miall, 1956). It has been shown (Stewart, 1948; Cochrane, 1960) that P.M.F. may progress after dust exposure has ceased. In simple pneumoconiosis, however, it is believed that this does not occur and that the development of the condition can be regarded as a continuous process, the amount of abnormality revealed in the radiograph increasing with increasing amounts of dust retained in the lungs.

Unfortunately, it is not possible to obtain a precise measure of this continuous variation in radiological abnormality, in view of the inherent difficulties of reading the radiographs. The best that can be done is to subdivide the abnormality scale in terms of a number of discrete categories; the I.L.O. classification used in these surveys divides the continuum of abnormality into four intervals, denoted respectively categories $0,1,2$, and 3. As the abnormality value increases, the position of the film moves along the scale from the lower limit of category 0 to the upper, then over the boundary between categories 0 and 1 , across category 1 and so on, until the upper limit of category 3 is reached or P.M.F. intervenes. Thus any given category of simple pneumoconiosis covers a range of abnormality values; in particular, the upper part of category 0 includes some films with radiological abnormality just insufficient to attain the category 1 classification.

We have seen that if a given individual is exposed to airborne dust his radiological abnormality will, in the absence of P.M.F., tend to increase with increasing exposure and, starting from the lower limit of category 0 , will traverse each of the categories of simple pneumoconiosis in turn. The precise form of this abnormality-exposure relation will depend on the way in which these two quantities are measured, i.e. on the choice of the abnormality and exposure scales. From the point of view of any mathematical analysis the most convenient form of relationship is a straight line, and it is therefore desirable to choose the scales accordingly.

The classification of simple pneumoconiosis into categories $0,1,2$, and 3 is based on an arbitrary subdivision of the continuum of abnormality, and the category numbers have no arithmetical significance in this context. In other words, there is no reason to suppose that each category covers an equal part of the abnormality scale or that category 0 represents abnormality values between 0 and 1 on the scale, category 1 represents abnormality values between 1 and 2, and so on. The absence of any basic measure of abnormality means that we are free to choose any convenient scale. It can be shown mathematically that a scale which leads to a straight line abnormality-exposure relation always exists, provided only that abnormality increases with increasing exposure. When the abnormality scale is defined the position of the origin and the scaling factor are arbitrary, and in the analyses to be described the boundary between categories 0 and 1 is taken as zero on the scale, and the boundary between categories 1 and 2 as unity.

The choice of a suitable scale for the exposure variable is a more straightforward matter. As in many other similar applications (Finney, 1952) it is most convenient to work in terms of the logarithm of the exposure variable, denoted the "dosage". Thus, for any given individual, the abnormalityexposure relationship takes the form shown in Fig. 1.

It follows that the negative abnormality values correspond to category 0 , the values between 0 and 1 correspond to category 1 , and the values in excess of 1 correspond to category 2 , followed by category 3. Two of the category boundaries are arbitrary and the third boundary-between categories 2 and 3can only be determined by reference to the natural history of pneumoconiosis amongst human populations. The development of simple pneumoconiosis beyond a reasonably advanced stage (generally regarded as about the lower limit of category 2) may be complicated by the superimposition of P.M.F. (Cochrane, Davies, Chapman, and Rae, 1956). As this possibility cannot be excluded amongst the populations under examination it is not possible to determine the precise position of the boundary between categories 2 and 3 on the scale. From the point of view of the subsequent analysis this is not important, as the calculations are concerned only with the boundaries between categories 0 and 1 and between categories 1 and 2 .

For the particular individual in question, radiological abnormality $\left(y_{o}\right)$ and dosage $\left(x_{c}\right)$ are assumed to obey a linear relation of the form,

$$
y_{o}=\alpha_{o}+\beta_{o} x_{o},
$$

where $\alpha_{o}$ and $\beta_{o}$ are two parameters which characterize his response to the particular measure of exposure under consideration. In this context the parameter $\beta_{o}$ is necessarily positive, as it is assumed that abnormality must increase with increasing exposure.

When a population of subjects is exposed to a given hazard the corresponding individual responses will not be identical, because of the differences from man to man which exist in human populations. It is assumed that these individual variations may be 


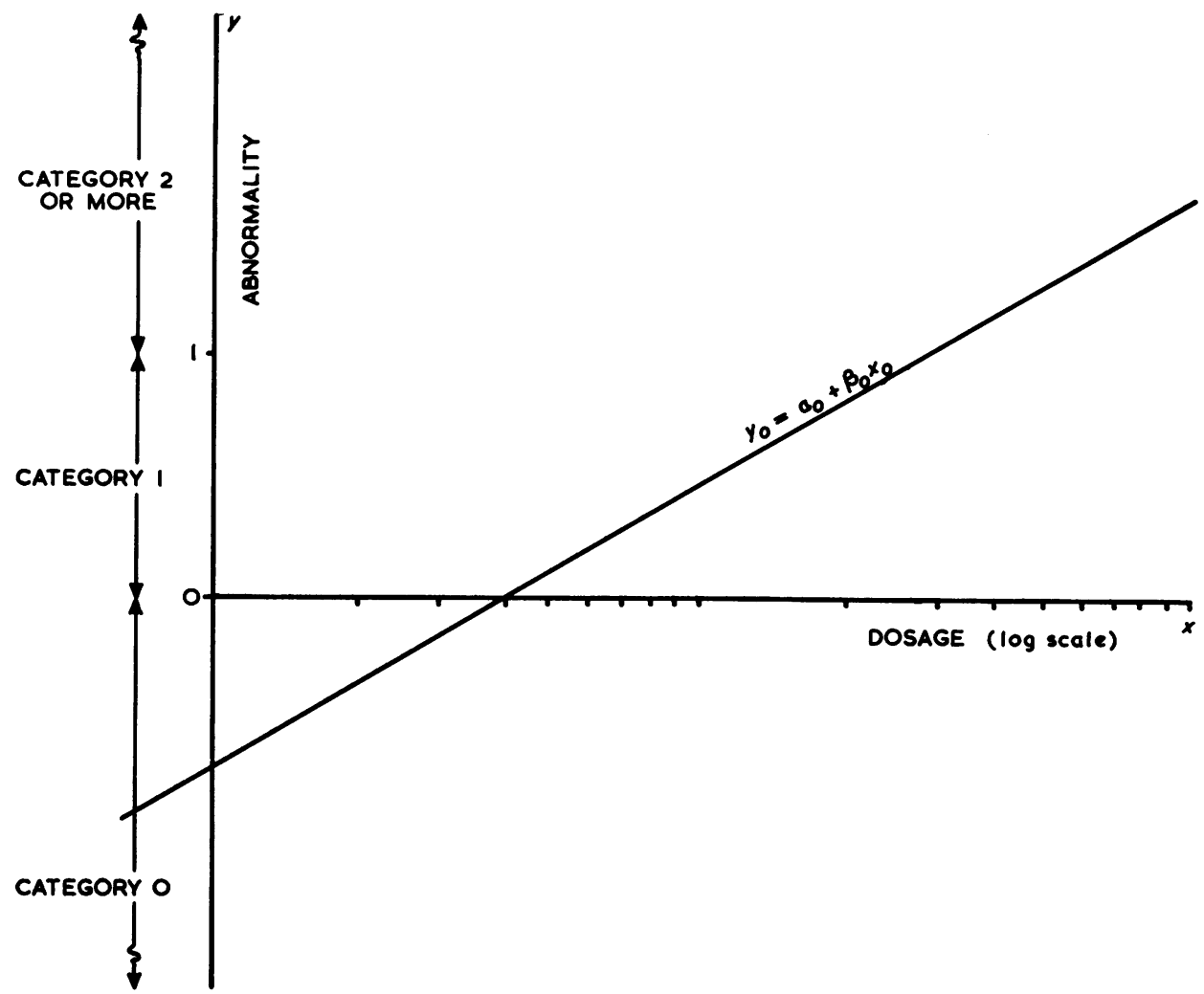

FIG. 1.-The abnormality-exposure relationship.

represented by corresponding variations in the value of the parameter $\alpha_{o}$, i.e. in the "basic" abnormality or susceptibility of the individual corresponding to a zero dosage [i.e. putting $x_{o}=0$ in equation (1)]. As in many other fields of application in which similar techniques are applied (Finney, 1952), it is assumed that the individual values of this parameter follow a Gaussian (normal) distribution, with mean $\alpha$ and variance $\sigma^{2}$. Thus, $\alpha$ can be regarded as the average value of $\alpha_{0}$ in the population and $\sigma^{2}$ is a measure of the variability of the abnormalityexposure relationship from individual to individual. It should be noted that $\beta$ is assumed to be constant throughout the whole population. This implies that the variability of the abnormality values $\left(y_{o}\right)$ is independent of the applied dosage.

The Effect of Errors of Measurement.-The above analysis is based on the assumption that the true values of abnormality and dosage are known exactly. In fact this is not so, and it is necessary to take account of the errors of measurement.

The measurement of abnormality depends on the subjective assessment of a chest radiograph, with all the attendant uncertainties both in technique and interpretation. Fay and Ashford (1960) have analysed the errors associated with the classification of radiographs for simple pneumoconiosis. Their treatment is based on the concept of an " $x$-ray reading" scale of radiological abnormality, which is chosen in such a way that the readings $\left(y^{\prime}\right)$ on any given film follow a Gaussian distribution about the true abnormality value $(y)$ with characteristic variance $\rho^{2}$, which is independent of $y$. This scale is defined so that the boundaries between categories 0 and 1 and categories 1 and 2 occur respectively at the points zero and unity. Although the $x$-ray reading scale has these two points in common with the "abnormality" scale defined above, there is no a priori reason why the two scales should be the same. Analyses of the data obtained on the medical surveys suggest, however, that the $x$-ray reading and abnormality scales may be regarded as coincident, at least in the vicinity of the " $0-1$ " and " $1-2$ " boundaries. Under this assumption Ashford (1959) has shown that the effect of unbiased reading errors 
is to increase the variance of the observed relationship between abnormality and exposure to $\left(\sigma^{2}+\rho^{2}\right)$, leaving the $\alpha$ and $\beta$ parameters unchanged. Very little is known about the errors associated with taking the radiograph, but what evidence is available within the Pneumoconiosis Field Research suggests that they may be incorporated with the reading errors, and thus tend to inflate the value of $\rho^{2}$ without leading to any appreciable change in the form of the error distribution.

The measurement of the dosage is also subject to error and the estimates applied in the analyses are assumed to follow a Gaussian distribution about the true values, with variance $\tau^{2}$. The effect of these errors (Ashford, 1959) is further to increase the variance of the observed relationship to $\left(\sigma^{2}+\rho^{2}+\right.$ $\beta^{2} \tau^{2}$ ), again leaving the $\alpha$ and $\beta$ parameters unchanged.

Thus, provided the errors of measurement are made without bias and follow a Gaussian distribution, their effect is merely to increase the apparent variability of the individual responses, without changing the basic abnormality-response relationship.

The Quantal Relationship.-The approach developed in the previous paragraphs amounts to the assumption that there exists a basic relation between the observed values of the abnormality $\left(y^{\prime}\right)$ and the dosage $\left(x^{\prime}\right)$ such that $y^{\prime}$ follows a Gaussian distribution with mean $\left(\alpha+\beta x^{\prime}\right)$ and effective variance $\sigma^{\prime 2}\left(=\sigma^{2}+\rho^{2}+\beta^{2} \tau^{2}\right)$. This means that for a given observed value of the dosage $\left(x^{\prime}\right)$ the observed abnormality values will follow a characteristic bellshaped Gaussian distribution, as illustrated in Fig. 2.

As we are dealing with a statistical distribution (i.e. as the man's abnormality must have some value, large or small), the total area under the Gaussian curve must be unity. If a man selected at random from the population is exposed to an observed dosage $\left(x^{\prime}\right)$ the probability that his observed abnormality $\left(y^{\prime}\right)$ will exceed any particular point $y_{i}$ on the scale is represented by the shaded area under the curve to the right of $y_{i}$. In mathematical terms,

$$
\text { Prob. } \begin{aligned}
\left(y^{\prime} \geqslant y_{i}\right) & =\int_{\left\{\frac{y_{i}-\overline{\alpha+\beta x^{\prime}}}{\sigma^{\prime}}\right\}}^{\infty} \phi(t) d t \\
& =\Phi\left\{\frac{\alpha+\beta x^{\prime}-y_{i}}{\sigma^{\prime}}\right\},
\end{aligned}
$$

where $\phi(t)$ and $\Phi(t)$ denote respectively the frequency function and the cumulative probability function corresponding to the standardized Gaussian distribution. Reference to Fig. 1 shows that the probability that the abnormality value will exceed the boundary between categories 0 and 1 (i.e. that the film will be read as category 1 or more) may be written,

$$
\text { Prob. }\left(y^{\prime} \geqslant 0\right)=\Phi\left\{\frac{\alpha+\beta x^{\prime}}{\sigma^{\prime}}\right\} \text {. }
$$

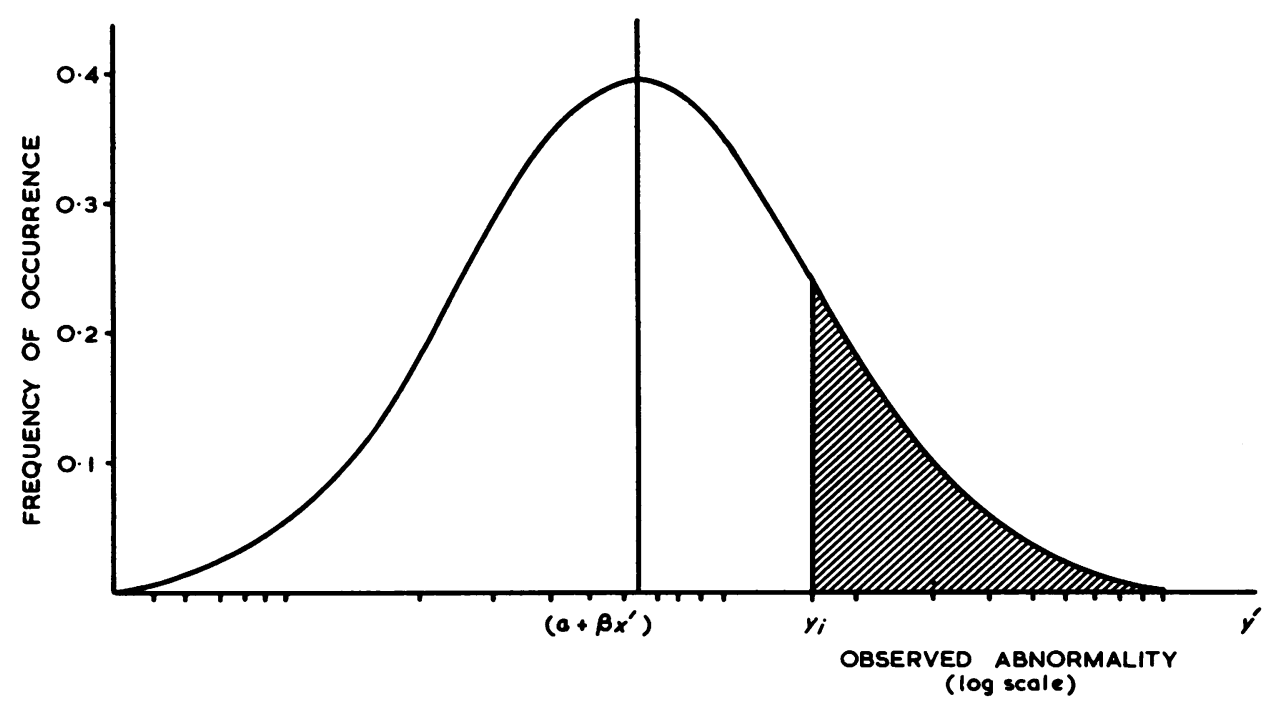

FIG. 2.-Distribution of abnormality for a given observed dosage. 


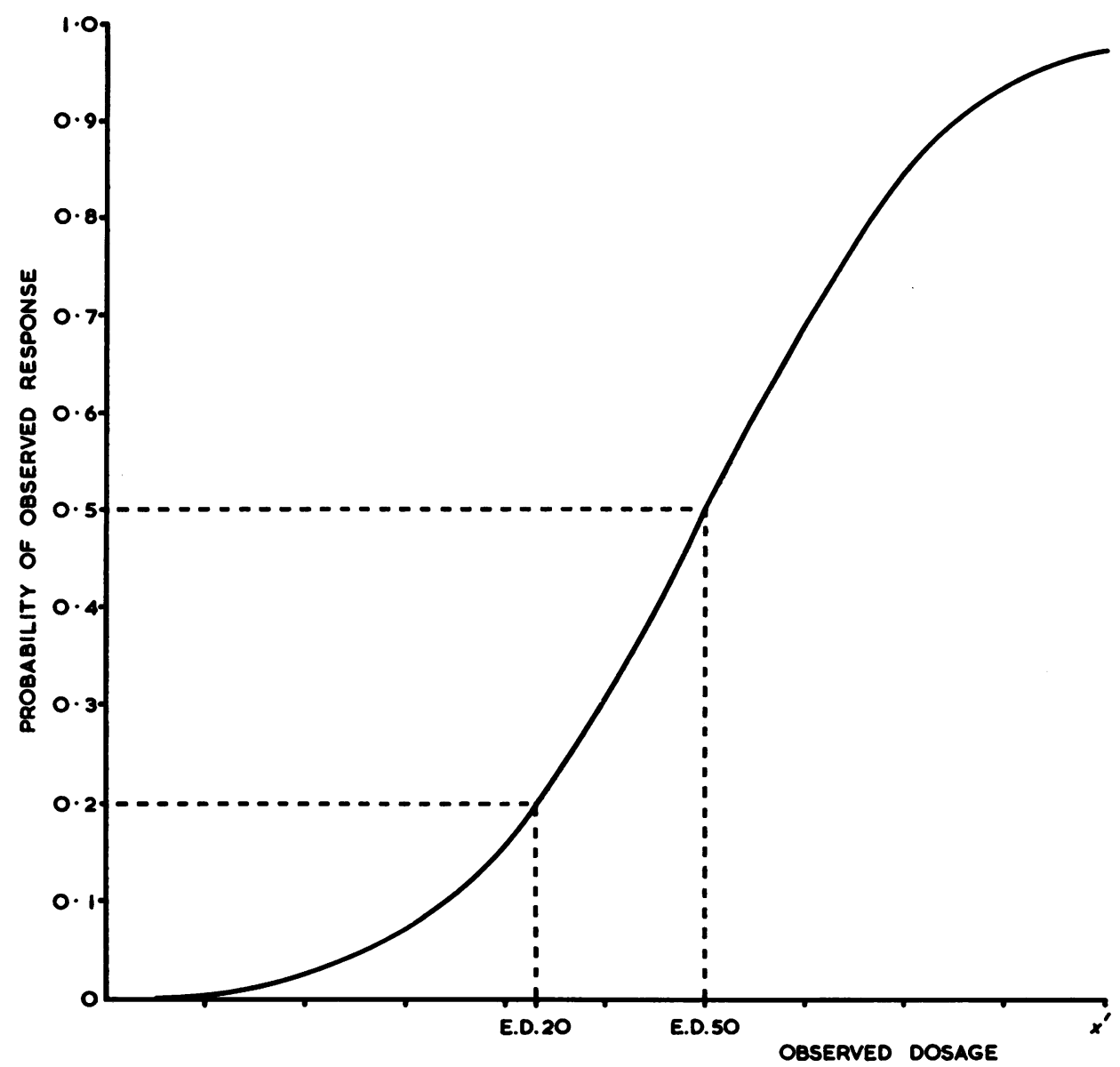

FIG. 3.-A typical sigmoid relationship.

Similarly the probability that the abnormality value will exceed the boundary between categories 1 and 2 (i.e. that the film will be read as category 2 or more) may be written,

$$
\text { Prob. }\left(y^{\prime} \geqslant 1\right)=\Phi\left\{\frac{\alpha+\beta x^{\prime}-1}{\sigma^{\prime}}\right\} \text {. }
$$

In practice the abnormality values can only be observed in terms of the discrete categories, i.e. in terms of the proportion of men with a given dosage who have developed a given category of pneumoconiosis or more. This means that the data on which studies of abnormality and dosage are based must take the form of the quantal relations (3) and (4), instead of the direct quantitative relation (1). Both the quantal relations involve the same basic parameters $\alpha, \beta$, and $\sigma^{\prime}$, and Ashford (1959) has shown how these may be estimated from the quantal data, and hence how the underlying quantitative relation may be deduced. Briefly, the method depends upon expressing the probabilities that a man's film is read as category 0 , category 1 , or category 2 or more, in terms of expressions (3) and (4). On this basis the likelihood of any given set of observations may be expressed as a function of the parameters $\alpha, \beta$, and $\sigma^{\prime}$, and the best estimates of the values of these parameters derived by the method of maximum likelihood.

Equation (3) relates the probability of the man's radiograph being classified as category 1 or more (i.e. of response) to the observed dosage. When the probability is plotted against dosage the relation takes the characteristic form of an S-shaped curve as shown in Fig. 3. This type of dosage-response curve is familiar in many branches of biological research, and Roach (1953) has referred to the 
possible application of the general method to the study of coalworkers' pneumoconiosis.

The probability of response increases smoothly from zero for small values of the dosage to unity for large values. The effective dosage (E.D.) values corresponding to a $20 \%$ and $50 \%$ probability of response are denoted respectively the E.D. 20 and E.D. 50, and these values are frequently used to provide an assessment of the relation.

For the purposes of the statistical analysis it is more convenient to work in terms of a linear relationship, and it is the accepted practice (Fechner, 1861; Bliss, 1935) to adjust the probability scale to achieve this effect, by means of the "probit" transformation. When this is done the probit* $(p)$ of the probability of response $(P)$ is related to the observed dosage as shown in Fig. 4. This relation between probit and dosage provides a convenient summary of the effect of exposure on abnormality and thus provides a measure of the hazard. As it is linear in form only two parameters (position and slope) are involved, and these reflect the three parameters $\alpha, \beta$, and $\sigma^{\prime}$ associated with the underlying abnormality-exposure relationship. Although the dosage-response relation

*This is defined by the relation: $-P=\Phi(p)$. shown in Fig. 4 is derived from the underlying abnormality-exposure relation given in Fig. 1, it must be emphasized that the former is concerned only with the chance of exceeding some given point on the abnormality scale, whereas the latter deals with the abnormality value itself.

For the category 1 or more response the linear relation takes the form;

$$
p=\frac{\alpha+\beta x^{\prime}}{\sigma^{\prime}} .
$$

The point at which this line crosses the $x^{\prime}$-axis $[\alpha / \beta]$ corresponds to a $50 \%$ probability of response, and is denoted the E.D. 50. The position of this point depends only on the position and slope of the underlying abnormality-exposure relation and is independent of the variability of the individual susceptibilities and of the errors of measurement. The slope of the quantal response line is $\beta / \sigma^{\prime}$ and is thus directly proportional to the slope of the underlying response line and inversely proportional to the effective variability of the individual susceptibilities. Any reduction in the errors of measurement or in the individual variation within the population would lead to a corresponding increase in the slope of the quantal response line. In the limit the

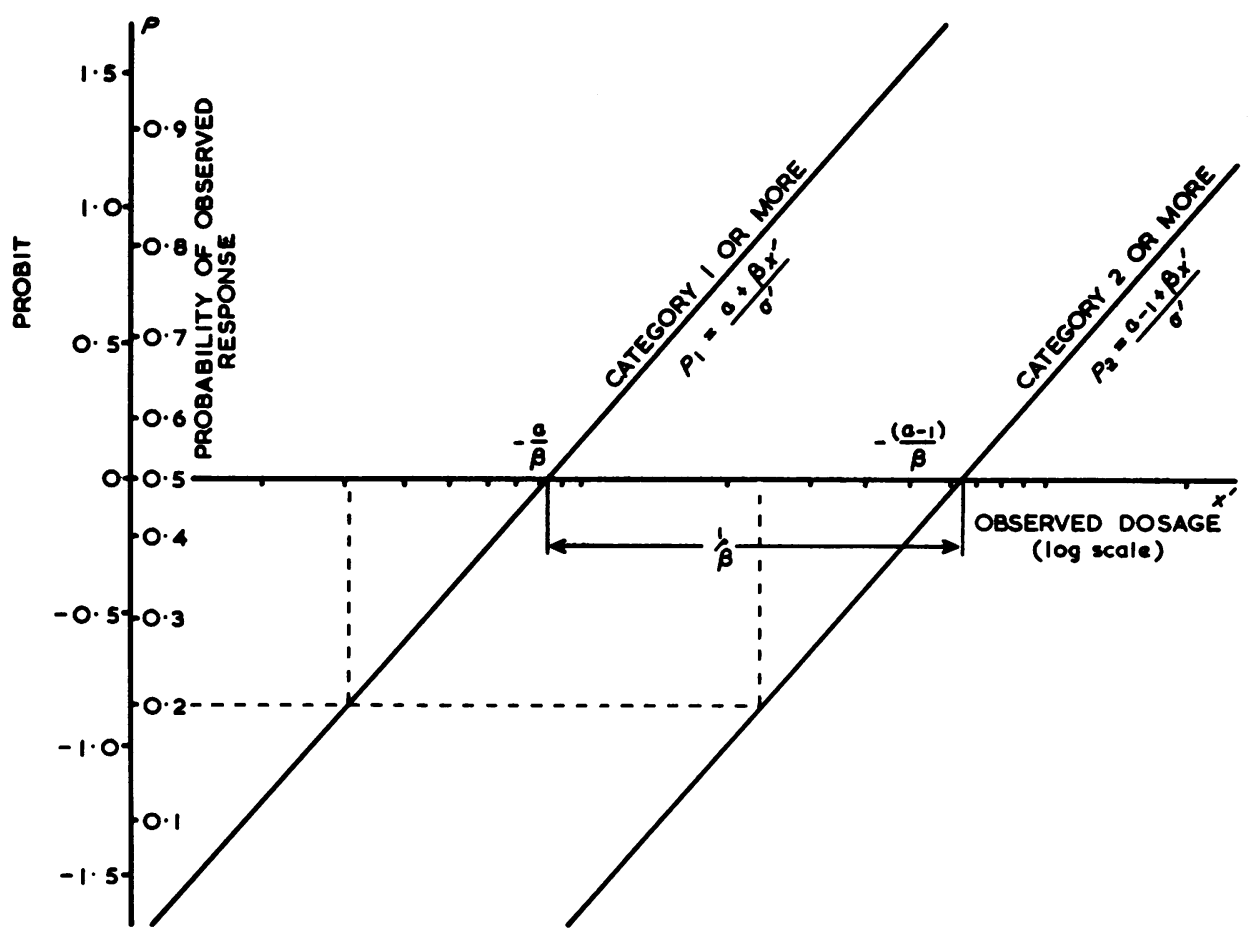

Fig. 4.-A typical probit relationship. 
quantal response line tends to become parallel to the $p$-axis as the effective variability tends towards zero. Under these conditions there would be a constant "threshold" dose for the whole population and the sigmoid curve illustrated in Fig. 3 would become a "step" function.

If we consider two different response levels say $P_{a} \%$ and $P_{b} \%$, the difference between the dosages corresponding to the E.D. $P_{a}$ and the E.D. $P_{b}$ will be, from (5),

$$
x_{a}-x_{b}=\frac{\sigma^{\prime}\left(p_{a}-p_{b}\right)}{\beta}
$$

and will thus depend on $\beta$ and $\sigma^{\prime}$, and not on $\alpha$. As the dosage is measured on a logarithmic scale this means that the ratio of the E.D. $P_{1}$ to the E.D. $P_{2}$ will depend on the effective variance $\sigma^{\prime}$, as well as on $P_{1}$ and $P_{2}$.

Reference to equations (3), (4), and (5) shows that the relation between the probit of the probability that the subject develops category 2 or more pneumoconiosis and dosage takes the form,

$$
p=\frac{\alpha-1+\beta x^{\prime}}{\sigma^{\prime}} .
$$

Thus the quantal response lines for category 1 or more and category 2 or more pneumoconiosis are parallel, the former lying to the left of the latter (Fig. 4). The horizontal difference between the two lines $(i . e$. the difference in dosage values corresponding to any given probability of response) is equal to the reciprocal of the slope of the underlying abnormality-dosage relationship, $1 / \beta$. As dosage is measured on a logarithmic scale the ratio of corresponding E.D's for category 1 or more and category 2 or more pneumoconiosis is therefore a constant for all response levels and all analyses dealing with the same population, regardless of the errors.

Measures of Exposure.-At the present time there are many uncertainties about which particular aspects of environmental exposure are of importance in the development of simple pneumoconiosis (Fay and Hicks, 1960). Indeed it will be one of the main tasks of the Pneumoconiosis Field Research to clarify the situation. In the absence of definite information about the nature of the hazard there are several possible measures of exposure which might be considered, each reflecting a different hypothesis about the relation between abnormality and exposure. Examples are (i) total particle number exposure in the 1-5 micron size range [expressed as the integrated product of average number concentration (all dust, 1-5 microns) and time at risk], (ii) total surface area exposure in the 1-5 micron size range, (iii) quartz exposure in terms of the surface area of particles in the $\mathbf{0 . 5}-5$ micron size range, and so on. Measures of exposure of this kind involve a detailed knowledge of the composition and concentration of the airborne dust particles throughout the whole of the working life of the man examined. This type of information is not available for the period before the first medical surveys at the selected collieries. It is therefore necessary to turn to less refined measures based on the men's working histories, in terms of the periods spent in particular occupations or types of environment, to represent the man's exposure up to the time of the first medical survey.

To illustrate the use to which the industrial history data may be put we will consider the prevalence of pneumoconiosis amongst a group of men who have worked for varying periods in one specific occupation. If the prevailing level of the particular type of hazard which is relevant to the causation of pneumoconiosis (denoted $l$ ) has remained constant throughout the whole period, the total exposure of a man who has followed the occupation for a time $t$ may be represented by the product $l \times t$. His "dosage" (i.e. the logarithm of his total exposure) will then be

$$
x=\log (l \times t)=\log l+\log t .
$$

Reference to equation (1) shows that the underlying dosage-abnormality relation takes the form,

$$
y=(\alpha+\beta \log l)+\beta \log t \quad .
$$

It follows that there is an equivalent abnormalitytime relationship, and that log time may be regarded as a substitute for the true dosage, which is unknown. Comparison of equations (1) and (9) shows that the slope of the corresponding response lines remains unchanged, although the constant term $\alpha$, which represents average susceptibility, is increased by a factor $\beta \log l$ (i.e. by a factor proportional to the logarithm of the mean exposure level).

Even if adequate quantitative and qualitative data are available about environmental conditions, the precise combination of these measurements which represents the true hazard is unknown. Analyses in terms of measured exposure must therefore be directed towards the testing of certain hypotheses about the nature of the hazard and it is unlikely that, on any one occasion, the hypothesis chosen will correspond exactly to the true situation. This means that, if a particular hypothesis $H$ is under investigation, the measurements of dosage $x_{H}$ based on this hypothesis will not correspond exactly to the true measurements of dosage $x$ expressed in the correct terms. For this reason the dosage values $x_{H}$ used in the analysis must be regarded as estimates of the true dosages $x$. On the assumption that the $x_{H}$ values follow a Gaussian 
distribution with mean $\left(x+\lambda_{H}\right)$ and variance $\tau_{H}{ }^{2}$, the underlying relation between abnormality and exposure (when expressed in terms of hypothesis $H$ ) will take the same form as the corresponding relation between abnormality and period of exposure given at equation (9), the only difference being that $\lambda_{H}$ is substituted for $\log l$ and the dosage $x_{H}$ is substituted for the logarithm of the time spent in the particular occupation. The abnormality-exposure relation when time is taken as a measure of exposure (amongst a group of men who have been exposed to only a single level of the hazard) is therefore equivalent to the relation when exposure is measured in terms of some particular hypothesis $H$, unless this hypothesis happens to provide an exact measure of the hazard (when both $\lambda_{H}$ and $\tau_{H}{ }^{2}$ will vanish).

For the type of analysis under consideration the values of the dosage $x_{H}$ are obtained by sampling methods and are not therefore known exactly. The estimated values $x_{H}^{\prime}$ are found to follow a Gaussian distribution about $x_{H}$ with variance $\tau_{H}{ }^{\prime 2}$. Thus, the effective variance of the $x_{H}^{\prime}$ about the true dosage $x$ will be $\left(\tau_{H}{ }^{2}+\tau_{H}{ }^{2}\right)$. If two hypotheses are compared, the better (i.e. the one which gives the closer approximation to the true hazard) will be the hypothesis associated with the smaller value of $\tau_{H}{ }^{2}$.

Comparison of Hazard Associated with Different Occupations.-If a population of men is assigned at random to one or other of two occupations in which they spend the remainder of their working lives up to the time of the medical examination, and if the mean levels of the hazard associated with the occupations are denoted $l_{a}$ and $l_{b}$, the corresponding abnormality-time relationships may be written,

$$
\text { and } \begin{aligned}
y_{a} & =\left(\alpha+\beta \log l_{a}\right)+\beta \log t_{a} \\
y_{b} & =\left(\alpha+\beta \log l_{b}\right)+\beta \log t_{b} .
\end{aligned}
$$

The variances of the individual abnormality values are respectively,

$$
\begin{aligned}
\sigma_{a}{ }^{2} & =\sigma^{2}+\rho^{2}+\beta^{2} \tau_{a}^{2} \\
\text { and } \sigma_{b}{ }^{2} & =\sigma^{2}+\rho^{2}+\beta^{2} \tau_{b}{ }^{2}
\end{aligned}
$$

where $\tau_{a}^{2}$ and $\tau_{b}^{2}$ are associated respectively with the variances of the errors in the $\log t_{a}$ and $\log t_{b}$ measurements. In general, $\tau_{a}{ }^{2}$ and $\tau_{b}{ }^{2}$ (and hence ${\sigma^{\prime}}_{a}^{2}$ and ${\sigma^{\prime}}_{b}^{2}$ ) will be different.

Equation (5) shows that the corresponding quantal relationships for category 1 or more pneumoconiosis are,

$$
\begin{gathered}
p_{a}=\frac{1}{\sigma_{a}^{\prime}}\left[\left(\alpha+\beta \log l_{a}\right)+\beta \log t_{a}\right] \\
\text { and } p_{b}=\frac{1}{{\sigma^{\prime}}_{b}^{\prime}}\left[\left(\alpha+\beta \log l_{b}\right)+\beta \log t_{b}\right] .
\end{gathered}
$$

The two lines will, in general, differ both in position (unless $l_{a}=l_{b}$ and $\tau_{a}=\tau_{b}$ ) and slope (unless $\tau_{a}=\tau_{b}$ ). Because the slopes are not necessarily the same the lines may cross, and comparisons between the relative hazards associated with the two occupations in terms of the quantal response lines themselves may be ambiguous. In other words, the usefulness of the quantal response lines for the comparison of the hazards associated with two different occupations is limited by the differential effect of errors in the dosage variable. On the other hand, the analysis in terms of the underlying quantitative response, to obtain estimates of $\beta, \sigma^{\prime}{ }_{a}, \sigma^{\prime}{ }_{b},\left(\alpha+\beta \log l_{a}\right)$, and $\left(\alpha+\beta \log l_{b}\right)$ [equations (10)-(13)] will provide direct information about the ratio $\left(l_{a} / l_{b}\right)$ of the levels of hazard associated with the two occupations.

A Typical Analysis.-An example of the application of the methods described above is provided by an analysis of the data obtained on the first medical survey at a particular colliery for a group of men who had previously worked only in a single specified occupation. To reduce the calculations to manageable proportions the 371 men concerned were divided into eight groups according to period spent in the occupation, and the numbers of men in each group whose radiographs were classified as category 0 , category 1 , and category 2 or more were recorded. In theory each individual could have been regarded as defining a separate group, but a considerable saving can be made in the calculations without any appreciable effect on the final results if the men with similar exposures are grouped together for the analysis. On the basis of the numbers of men with category 0 , category 1 , and category 2 or more pneumoconiosis in the various groups, the quantal relations between period spent in the occupation and the chance of developing category 1 or more and category 2 or more pneumoconiosis were calculated, and the underlying abnormalityexposure relation was derived.

In order to test whether the observed data are consistent with the assumptions on which the calculations are based, the expected numbers of men assigned to the various radiological categories (predicted on the basis of the estimated abnormalityexposure relation) were compared with the corresponding observed results. Details are given in the Table which shows that agreement between theory and observations is good. The $\chi^{2}$ value, after grouping the first three and last two entries in the Table, is 4.62 , which for 7 degrees of freedom is not significant $(\mathrm{p} \approx 0.7)$.

The corresponding quantal and quantitative relations are illustrated respectively at Figs. 5a and b. It should be noted (Fig. 5a) that the quantal response lines corresponding to category 1 or more and 
TABLE

PERIOD OF EXPOSURE AND PREVALENCE OF PNEUMOCONIOSIS AMONGST A GROUP OF COAL-MINERS WHO HAVE WORKED PREDOMINANTLY IN THE SAME OCCUPATION

\begin{tabular}{|c|c|c|c|c|c|c|}
\hline \multirow{3}{*}{$\begin{array}{l}\text { Mean Period } \\
\text { Spent (years) }\end{array}$} & \multicolumn{6}{|c|}{ Number of Men } \\
\hline & \multicolumn{2}{|c|}{ Category 0} & \multicolumn{2}{|c|}{ Category 1} & \multicolumn{2}{|c|}{ Category 2 or More } \\
\hline & Observed & Expected & Observed & Expected & Observed & Expected \\
\hline $\begin{array}{r}5.8 \\
15.0 \\
21.5 \\
27.5 \\
33.5 \\
39.5 \\
46.0 \\
51.5\end{array}$ & $\begin{array}{l}\left.\begin{array}{r}98 \\
51 \\
34 \\
35\end{array}\right\} 183 \\
32 \\
23 \\
\left.\begin{array}{r}12 \\
4\end{array}\right\} 16\end{array}$ & 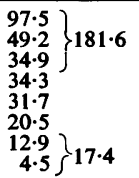 & 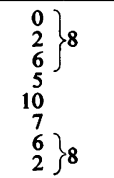 & 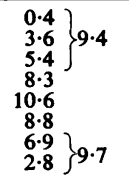 & $\left.\begin{array}{r}0 \\
1 \\
3 \\
8 \\
9 \\
8 \\
10 \\
5\end{array}\right\} 15$ & $\left.\begin{array}{l}\left.\begin{array}{l}0 \cdot 1 \\
1 \cdot 3 \\
2 \cdot 7 \\
5 \cdot 5\end{array}\right\} 4 \cdot 1 \\
8 \cdot 7 \\
8 \cdot 7 \\
8 \cdot 2 \\
3.8\end{array}\right\} 12 \cdot 0$ \\
\hline
\end{tabular}

category 2 or more pneumoconiosis are parallel, as required by the theoretical treatment. For this reason it is possible to obtain more reliable estimates of the quantal relations than could be derived if the corresponding relations for category 1 or more and category 2 or more pneumoconiosis were calculated separately. A further advantage as compared with the conventional quantal response analysis is that the underlying relation between abnormality and exposure provides a better description of the natural history of simple pneumoconiosis and enables conclusions to be drawn about the chance of developing any specified degree of pneumoconiosis, whether or not it forms the boundary of two of the standard

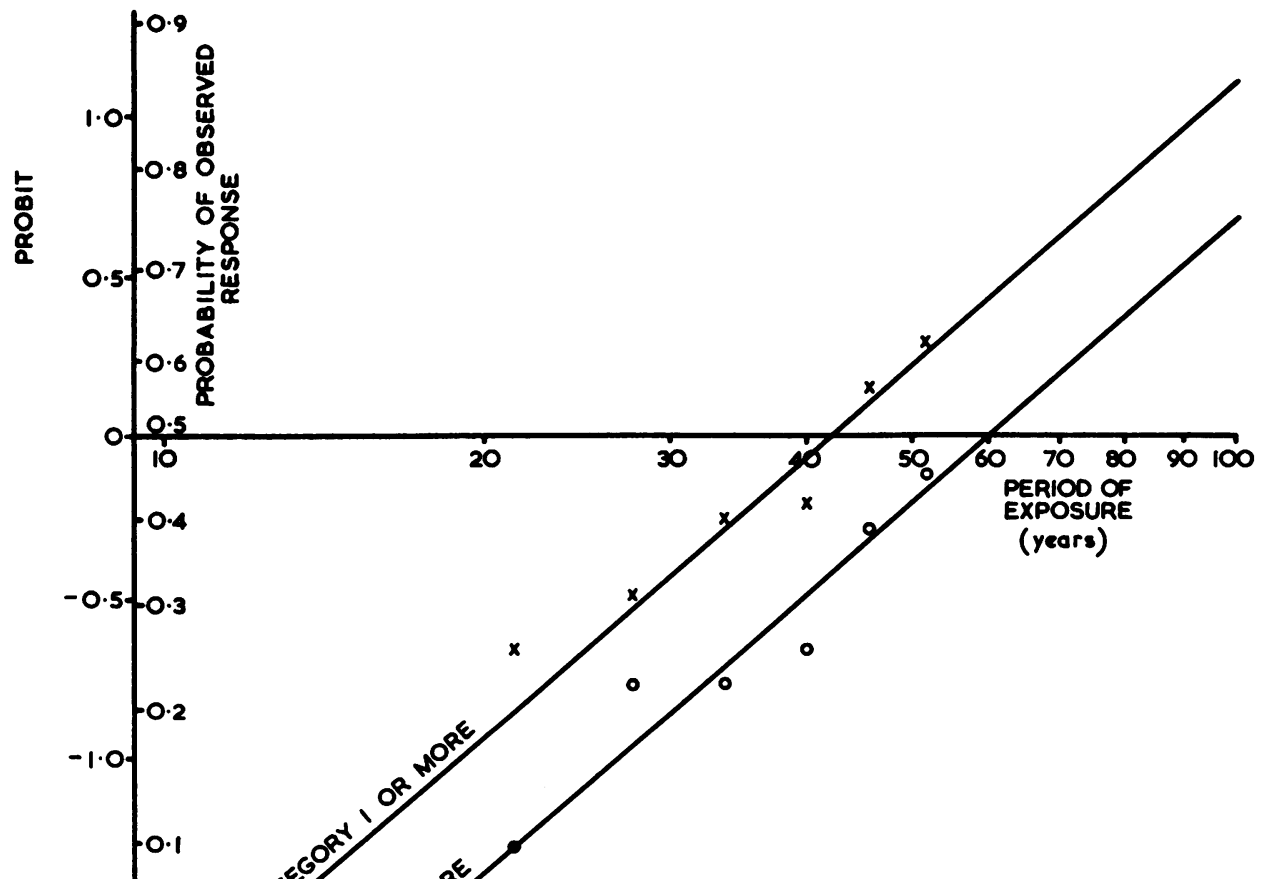

FIG. 5a

Fig. 5.-Analysis of the prevalence of pneumoconiosis amongst a group of men who have worked only in one particular occupation. (a) The quantal response; (b) (Opposite) The quantitative response. 
categories. For example, for a period of exposure of 20 years it is found that the corresponding observed abnormality value $y^{\prime}$ will follow a Gaussian distribution, with mean -1.52 (Fig. 5b) and standard deviation 1.56. Thus by reference to tables of the Gaussian distribution it can be deduced that the chance of the abnormality value exceeding zero (i.e. of the man being classified as category 1 or more) is approximately one-sixth. The new approach also provides an estimate of the combined variability of the individual susceptibilities and the errors of measurement; in this particular analysis we have seen that the calculated value of the standard deviation of the combined error is 1.56 .

\section{The Effect of "Mixed Exposures"}

Method of Approach.-The approach described above provides a satisfactory basis for estimating the relation between abnormality at the time of the first medical survey and previous environmental exposure, subject to the proviso that this latter measurement can be made in terms of a single variable (i.e. that the man has worked only in one occupation or class of environment). Unfortunately, it is usual in the mining industry for men to follow a variety of different jobs during the course of their working lives, thereby being exposed to a number of different levels of hazard. It is the normal practice for a new recruit to start work on the surface, or at non-facework underground, and to progress after a period (depending on local custom and circumstances) to the more highly paid occupations on the coal-face. Generally speaking, a coal-face worker will continue to work on the coal-face until the decline of his physical powers as a result of increasing age or of other causes makes it necessary for him to transfer to less arduous (and less remunerative) employment away from the coal-face, either underground or on the surface.

When the analysis of the results of the first round of medical surveys was carried out it would have been most unfortunate to have had to exclude the large proportion of men with "mixed" industrial histories. Consideration was therefore given to the development of a method of analysis to cope with the situation in which the man's past environmental exposure must be measured in terms of the periods spent in two or more specific occupations or classes of environment. Following the method of approach described above for dealing with exposure at a single

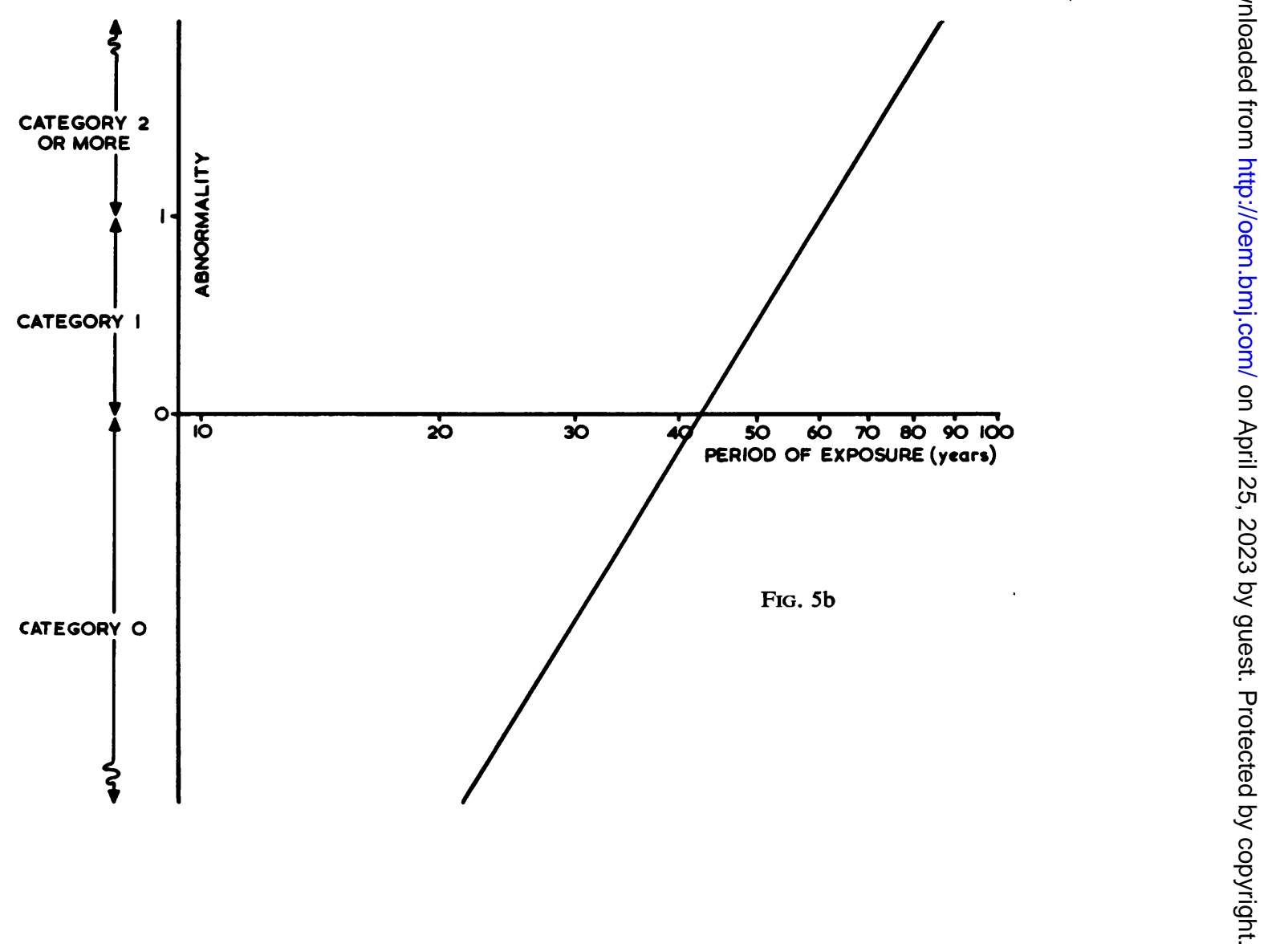


level of the hazard, we will assume that a man has spent periods $t_{1}$ and $t_{2}$ in two distinct occupations or classes of environment $E_{1}$ and $E_{2}$. The prevailing levels of the hazard (assumed to be constant) are denoted $l_{1}$ and $l_{2}\left(=k l_{1}\right)$. Thus, environment $E_{2}$ is assumed to be $k$ times as hazardous as environment $E_{1}$. The man's total exposure will consist of the sum of his exposures in each of the two environments separately. In these circumstances his total dosage (i.e. the logarithm of his total exposure) will be given by the expression,

$$
\begin{aligned}
x & =\log \left(t_{1} l_{1}+t_{2} l_{2}\right) \\
& =\log l_{1}+\log \left(t_{1}+k t_{2}\right)
\end{aligned}
$$

The abnormality-exposure relation will take the form,

$$
y=\left(\alpha+\beta \log l_{1}\right)+\beta \log \left(t_{1}+k t_{2}\right) .
$$

Comparison with equation (9), which describes the corresponding relation for exposure in a single environment, shows that the effective dosage can be taken as $\log \left(t_{1}+k t_{2}\right)$. It follows that the variance of the Gaussian distribution of the observed individual responses is given approximately by the expression,

$$
\sigma^{\prime 2}=\sigma^{2}+\rho^{2}+\beta^{2}\left[\frac{t_{1}{ }^{2} \tau_{1}{ }^{2}+k^{2} t_{2}{ }^{2} \tau_{2}{ }^{2}}{\left(t_{1}+k t_{2}\right)^{2}}\right],
$$

where $\tau_{1}^{2}$ and $\tau_{2}^{2}$ are the variances associated with the measurements of the individual dosages $\log t_{1}$ and $\log t_{2}$.

By means of equations (17) and (18) the quantal response relationships based on the underlying quantitative response can be expressed in terms of a "multi-dimensional" exposure $\left(t_{1}, t_{2}\right)$ in the same way as is done for a single-dimensional exposure. Ashford (1958a) has shown how an approach of this type may be applied to obtain information, from the "mixed" exposure data, about the dosageabnormality relation associated with each of the constituent hazards separately. In this connexion it should be noted that the presence of errors in the estimates of the time spent in the various classes of environment (which leads to the expression (18) for the effective variance of the individual responses) means that the quantal relationships between the probability of response and the multi-dimensional exposure cannot be derived directly from the quantal relations between the probability of response and each of the dimensions of exposure separately.

Applications.-Analysis of the results of the first round of medical surveys at the selected collieries (Hicks et al., 1961) has shown that there are, in general, four main types of environment which involve an appreciable pneumoconiosis hazard, namely hard heading (i.e. tunnelling in rock), coalface (coal-getting shift), coal-face (preparation shift), and elsewhere underground. The hazard associated with work on the surface can, in general, be regarded as negligible. It is also found that only a very small proportion of the men examined have had experience of hard heading and, if these men are excluded from the analysis, each man's exposure can be regarded as a three-dimensional variable corresponding to the periods spent in each of the three remaining environments.

Thus, if a man has spent $t_{1}$ years on the coal-face (coal-getting shift), $t_{2}$ years on the coal-face (preparation shift) and $t_{3}$ years elsewhere underground, his total exposure may be summarized in terms of the three-dimensional variable $\left(t_{1}, t_{2}, t_{3}\right)$. In this particular application of the multi-dimensional method (although this is not necessarily so in general) all periods of exposure in a given environment are taken together, whether or not they refer to the same working places or even to the same colliery. This approach involves the tacit assumption that each separate environment is associated with the same level of hazard for all working places. This assumption can be justified as a reasonable working approximation for a number of reasons. In the first place it is found that the majority of men have worked only at the colliery at which they were examined or at other collieries in the immediate vicinity. Secondly, studies carried out by the Pneumoconiosis Field Research have shown that conditions do not, in general, vary greatly for the same environment (a) from colliery to colliery in the same locality, (b) from one working place to another within the same colliery, or (c) from time to time at the same working place, provided that the methods of working are similar. Thirdly, the greater part of the men's exposure before the first medical surveys, took place during a period when methods of working were relatively stable, before the introduction of power loading on an appreciable scale. The final test of the validity of the assumption may be obtained by comparing the observed numbers of men with the various categories of pneumoconiosis with the numbers predicted on the basis of the assumed model; for a large number of analyses the agreement has been found to be extremely good (Hicks et al., 1961), and it can be asserted with confidence that the observed data are consistent with the theoretical treatment.

At one particular colliery the periods spent by each man (excluding those who had ever worked as hard headers) on the coal-face (coal-getting shift), coal-face (preparation shift), and elsewhere underground were calculated from his past working history. In order to reduce the magnitude of the mathematical calculations, the population (of some 1,700 men) was subdivided into about 50 different 
groups, each group including men with similar periods of exposure in each of the three main classes of environment. On the basis of the numbers of men in each group with category 0 , category 1 , and category 2 or more and the corresponding average periods of exposure in the three environments, the quantal response lines for category 1 or more pneumoconiosis and category 2 or more pneumoconiosis and the corresponding underlying abnormality-exposure relations were calculated for each class of environment separately. The mathematical calculations involved have been described in detail by Ashford (1958a; 1959). The resulting quantal response lines are shown at Fig. $6 \mathrm{a}$ and the under- lying quantitative relationship appears at Fig. $6 \mathrm{~b}$.

Fig. 6 a shows that, as required by the model, the quantal response lines from category 1 or more and category $\mathbf{2}$ or more pneumoconiosis for each of the environments separately are parallel. When the individual category 1 or more lines are considered, however, comparisons of the hazard associated with the three environments are ambiguous in the sense that, for example, work elsewhere underground appears to have been more hazardous for short periods of exposure and less hazardous for long periods of exposure than work on the coal-face. This ambiguity is a reflection of the fact (discussed above) that the errors associated with the use of the

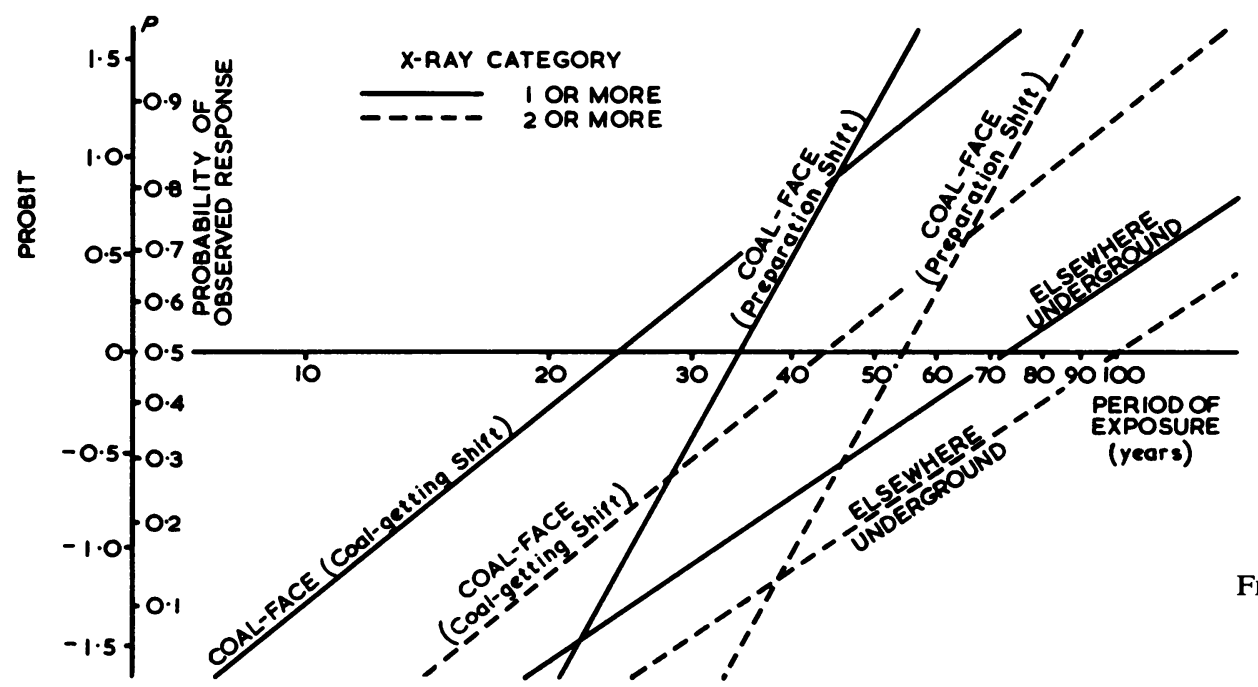

FIG. 6a

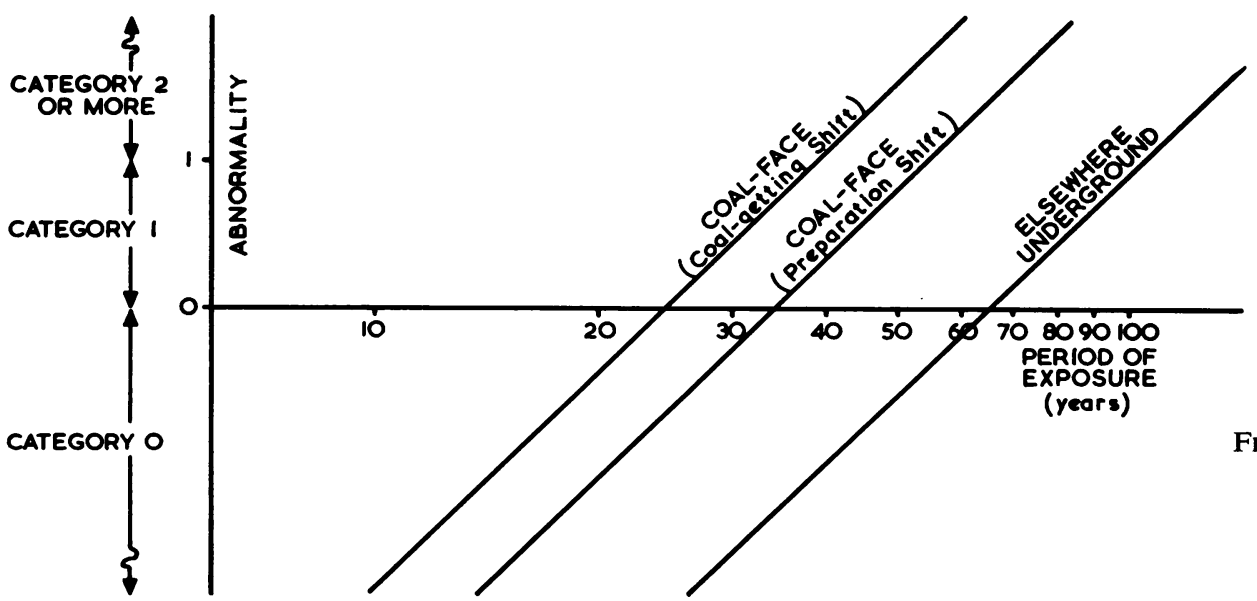

Fig. 6b

Fig. 6.-Multi-dimensional analysis of the prevalence of pneumoconiosis in a colliery population in terms of the periods spent on the coal-face (coal-getting shift), coal-face (preparation shift), and elsewhere underground. (a) The quantal 
period of exposure in any given environment as a measure of the hazard differ from environment to environment and that, in consequence, the observed quantal response lines are not, in general, parallel.

Consideration of the underlying abnormalityexposure relations enables the effect of the errors to be treated separately (Fig. 6). It will be seen that the abnormality-exposure lines corresponding to the three environments separately are parallel, as required by the method of analysis, and that the ambiguity associated with comparisons of the relative hazards in terms of the quantal response lines is now removed. As exposure is measured on a logarithmic scale, the ratio of the periods spent in any pair of the three environments leading to any particular abnormality value is constant and corresponds to the relative hazard. In this analysis the hazard on the coal-face (coal-getting shift) was about four-thirds of that on the coal-face (preparation shift) and about eight-thirds of that for exposure elsewhere underground. The standard deviations of the observed susceptibilities (which represent the summation of the variability associated with the true distribution of susceptibilities, the errors of taking and reading the radiographs, and the errors associated with the use of time as a measure of exposure) were respectively $1.5,0.72$, and 1.8 for the coal-face (coal-getting shift), coal-face (preparation shift), and elsewhere underground. These differences are a reflection of differences between the three environments in the errors associated with the use of time as a measure of exposure.

To summarize, the multi-dimensional approach has provided the means of estimating the past hazard associated with each of the three main environments separately, on the basis of the mixed working history data from all the men examined at a colliery, excluding only the small number of men who have worked as hard headers. This is only one of a number of applications of the method, which has also been used (a) to compare the hazards associated with two different occupations on the basis of groups of men who have worked mainly in one or other or both of the two occupations, (b) to compare the hazard associated with the colliery at which the men were examined with the average hazard at the other collieries at which they have worked in the past, on the basis of the corresponding periods spent in all dusty occupations, and (c) to analyse the data from successive medical surveys, as described below.

\section{Analysis of the Progression Data}

The Measurement of Progression.-Each successive round of medical surveys will provide information about the man's radiological appearance at the corresponding point in time. The classification of simple pneumoconiosis is made in terms of a small number of discrete categories, and inevitably each category covers a considerable range of abnormality. Hence, it is not possible to obtain any useful measure of progression merely by subtracting the "earlier" from the "later" reading, as would be the case if the measurements of abnormality could be made with precision on a continuous and uniform scale. There is the further complication that the radiograph readings are subject to error, which means that a man may be assigned to a higher category on the earlier survey than on the later one, if the readings are made independently.

The possibility of making use of the "average" of a number of independent readings to obtain a more reliable subdivision of the abnormality scale has been under active consideration since the start of the Research. There are, however, several inherent difficulties. In the first place the number of different sets of readings which can be obtained on each film is limited by the effort available; this point is particularly important when upwards of 30,000 men are involved. On the first round of medical surveys three such sets of readings were obtained at most. By averaging these three readings, each category can be divided into three (not necessarily equal) parts, but these sub-categories themselves cover a considerable range of abnormality. With the comparatively small amount of progression which has so far been found between the first and second medical surveys this subdivision would by no means provide an adequate substitute for a continuous scale. Secondly, a detailed study of the effect of "averaging" (Fay and Ashford, 1960) has shown that the position of the sub-categories so obtained depends on the errors associated with the individual sets of readings, and Ashford (1960) has shown that the magnitude of these errors may vary widely from one set of readings to another. In theory, a suitable correction for the individual reading errors could be applied, but the necessary calculations would be so complex as to cancel out the apparent simplicity of the method.

An alternative approach was therefore considered. This involved the examination of all the films obtained for each man (on successive surveys) at the same time; if any change in the characteristic abnormalities associated with simple pneumoconiosis was observed (after due allowance had been made for radiographic technique) the man was to be classified as showing evidence of "progression" (or "regression"). The fundamental disadvantages of this procedure are, first, that no quantitative information can be obtained of the change in abnor- 
mality associated with a "progression" (or "regression") reading and, secondly, that there is no certainty that any given amount of radiological change will be equally evident at all points on the abnormality scale.

For these reasons it was decided to consider the problem in terms of the changes (if any) in radiological category between the man's first exposure to airborne dust (when it is reasonable to assume that his radiological abnormality value will be at the lower limits of category 0 ) and the times of the successive medical surveys. This approach demands a knowledge of the man's radiological category at each medical survey, so that the position of his corresponding radiological abnormality value relative to the various category boundaries can be determined. The definitive readings have been shown (Ashford, 1960) to be free from systematic bias and therefore provide a satisfactory measure of radiological category for this purpose. There are two possible general procedures for obtaining the definitive radiological readings on a series of films taken at different points in time. On the one hand the films for successive surveys may be read independently, or, alternatively, the corresponding films on successive surveys may be examined side-by-side, the reading of each film being subject to influence by the appearance of the others. At the present time the relative merits of the two methods are being assessed within the Research, by means of a series of film reading trials.

The Measurement of Exposure.-If a man starts at the lower limit of category 0 on entering the mining industry, his radiological appearance at any given time will depend on his subsequent exposure. We have seen that each man's exposure at the time of the first medical survey may be expressed as a multi-dimensional variable corresponding to the periods he has spent in each of the main classes of environment. At the time of the second medical survey the man has been subject to a further ("measured") exposure, and his cumulative exposure since joining the mining industry can also be regarded as a multi-dimensional variable in terms of the periods spent in the main classes of environment up to the time of the first survey, with one or more additional dimensions corresponding to his "measured" exposure between the first and second surveys. For example, the man's total exposure up to the time of the second medical survey might possibly be represented by a four-dimensional variable, in terms of the periods spent before the first survey on the coal-face (coal-getting shift), coal-face (preparation shift), and elsewhere underground, and of his "measured" exposure between the two surveys. It has been shown that the use of the period of exposure in a given occupation as a substitute for true exposure is based on the assumption that the dosage corresponding to a period $t$ spent in an occupation with mean hazard $l$ is given by the expression,

$$
x=\log (l \times t)=\log l+\log t .
$$

If the mean levels of hazard associated with the three types of environment are denoted $l_{1}, l_{2}$, and $l_{3}$, and if the periods spent in these environments up to the time of the first medical survey are denoted $t_{1}, t_{2}$, and $t_{3}$ respectively, the man's dosage before the first medical survey is therefore,

$$
x=\log \left[\left(l_{1} \times t_{1}\right)+\left(l_{2} \times t_{2}\right)+\left(l_{3} \times t_{3}\right)\right] .
$$

His "measured" component of exposure, denoted $y$, between the first and second surveys will take the form of the sum of a series of products (corresponding to each of the occupational groups to which the man has belonged) of the mean hazard (expressed in accordance with the particular hypothesis about exposure which is under examination) and the corresponding period of exposure. If the levels of hazard $l_{1}, l_{2}$, and $l_{3}$ are expressed in the same form as the levels used for the calculation of the "measured" exposure, all four components of the man's exposure up to the time of the second survey will be expressed in the same terms. The man's total dosage then takes the form,

$$
x=\log \left[\left(l_{1} \times t_{1}\right)+\left(l_{2} \times t_{2}\right)+\left(l_{3} \times t_{3}\right)+y\right] .
$$

At first sight, the use of a multi-dimensional variable expressed in terms of a mixture of time and "measured" exposure might appear to be subject to criticism, but reference to expression (20) shows that in fact like is being added to like, and it therefore follows that the procedure is valid.

It can also be shown that the variance of the Gaussian distribution of individual exposures is given approximately by the expression,

$\sigma^{\prime 2}=\sigma^{2}+\rho^{2}+\beta^{2}\left[\frac{l_{1}{ }^{2} t_{1}{ }^{2} \tau_{1}{ }^{2}+l_{2}{ }^{2} t_{2}{ }^{2} \tau_{2}{ }^{2}+l_{3}{ }^{2} t_{3}{ }^{2} \tau_{3}{ }^{2}+y^{2} \tau^{2}}{\left(l_{1} t_{1}+l_{2} t_{2}+l_{3} t_{3}+y\right)^{2}}\right]$,

where $\tau_{1}^{2}, \tau_{2}^{2}, \tau_{3}^{2}$, and $\tau^{2}$ are respectively the variances associated with the measurements of $\log t_{1}, \log t_{2}, \log t_{3}$, and $y$ respectively. It should be noted that the variance $\tau^{2}$ of the "measured" component of dosage represents a summation of the errors of estimation associated with the particular measure of hazard chosen and of the errors associated with the choice of this particular measure of hazard as a representation of the man's true exposure.

By means of the underlying abnormality-exposure relation (1) and expressions (19), (20), and (21) the 
probability that a man has developed any given category of simple pneumoconiosis or more at the time of each successive medical survey may be expressed in terms of the two sets of multidimensional variables. Hence, the underlying abnormality-exposure relations for each of the dimensions separately may be estimated, and the effect of the "measured" component of exposure may be assessed. The relative accuracy of the resulting estimates will depend on the proportion of the man's total exposure which is accounted for by the "measured" component. The information about the relation between abnormality and the "measured" component of exposure will therefore become progressively more precise as the number of surveys increases.

An Example of the Method.-The proposed method may be illustrated by the analysis of the data obtained at one particular colliery between the first and second medical surveys. On this occasion the radiographs obtained on the surveys were classified independently and, by reading a check sample of first survey films again on the second survey, it was established that there had been no significant change in the levels of classification of the definitive readings.

The population considered for the progression analysis consists of all men examined on both first and second medical surveys with no record of exposure to airborne dust other than at the colliery during the intervening period. The following data are available for each man:

(i) Independent definitive readings of his chest radiographs on the two successive surveys, in terms of the I.L.O. (1953) classification;

(ii) Summary of his working history up to the time of the first medical survey, in terms of the periods spent in the main classes of general environment;

(iii) An unbiased estimate, of known accuracy, of his dust exposure between the two surveys.

For the purposes of this analysis it was decided that the working histories could best be represented in terms of the period spent in "dusty" occupations. This index of the past hazard was preferred to the more complex representation in terms of the three main classes of general environment discussed above, in view of the comparatively small number of men involved and of the small amount of radiological change reported. In any event it is found (using the methods described in Section 4) that the period spent in "dusty" occupations provides an adequate representation of the past hazard and correlates well with the prevalence of pneumoconiosis at the time of the first medical survey.
For this particular analysis it was decided to express the "measured" component of exposure as a single-dimensional variable, in terms of the product of total number count in the 1-5 micron size range and period of exposure; other measures of hazard could, of course, have been calculated on the basis of the information available, depending on the particular hypothesis to be tested. As a result of the environmental sampling programmes carried out by Pneumoconiosis Field Research a "shift exposure index" is calculated for each sampling shift, in terms of the product of average dust concentration (p.p. ml. ${ }^{*}, 1-5$ microns) and time at risk (hours). The average exposure index for all shifts assigned to each occupational group is then calculated and a man's exposure in the group is obtained as the product of the "group exposure index" and the period spent in the group (for this purpose measured in months). The man's total exposure between the two surveys is obtained by taking the sum of the individual components corresponding to each of the occupational groups to which he has belonged during this period. The units of exposure used in these calculations are thus p.p. ml. (1-5 microns) $\times$ hours $\times$ months. For example, a figure of 75,000 exposure units could correspond to a period of 10 months spent in an environment where the duration of the working shift was seven and a half hours and the average concentration was 1,000 p.p. ml. (1-5 microns).

When the readings obtained on the first and second surveys are compared there are nine possible sequences of pairs of readings in terms of crossing the boundaries between categories 0 and 1 and categories 1 and 2: $(0,0),(1,1),(2$ or more, 2 or more) [no change]; $(0,1),(0,2$, or more $),(1,2$, or more) [apparent progression]; $(1,0),(2$ or more, 0$)$, and ( 2 or more, 1) [apparent regression]. Any apparent regressions are assumed to be due to variations in taking or reading the films and due account is taken of them in the analysis. Although the possibility of true regression of simple pneumoconiosis cannot be entirely ruled out it is considered to be unlikely on the evidence available (Cochrane, 1960). Using the methods which have been described the probability that any of these pairs of readings is obtained may be expressed as a function of the two multi-dimensional dosage variables.

The 485 men considered in the analysis were divided into about 50 different groups, each containing men with similar periods in dusty occupations before the first survey and "measured" exposures. In each group the numbers of men corresponding

\footnotetext{
*Particles per millilitre.
} 
to the nine pairs of first and second survey readings were recorded. There was a considerable amount of movement from category to category between the two surveys, although the overall progression was small. If all readings of category 2 or more are taken together (as they are in the analysis), a total of 57 men was assigned to a higher category on the second survey than on the first (apparent progression) and 43 men were assigned to a lower category (apparent regression). In view of the relatively high initial prevalence and the small overall change in radiological classification between the two medical surveys, it is apparent on general grounds that the "measured" exposure accounts for only a small proportion of the total hazard experienced by the men concerned. Hence, the accuracy of the resulting estimates of the relation between abnormality and "measured" exposure will be expected to be low.

On the basis of the numbers of men in the various groups assigned to categories 0,1 , and 2 or more on the two surveys and the corresponding periods spent in dusty occupations and "measured" exposures, an estimate was obtained of the abnormalityexposure relation associated with the "measured" exposure separately. [The basic mathematical procedures have been described in detail elsewhere (Ashford et al., 1960)]. Because of the small amount of radiological change certain simplifying assumptions were made in this particular analysis, as the complete (and more time consuming) treatment of the data would not have been worthwhile. The resulting quantal response lines for "measured" exposure corresponding to category 1 or more and category 2 or more pneumoconiosis are shown in Fig. $7 \mathrm{a}$, and the corresponding quantitative abnormality-exposure relation appears at Fig. $7 \mathrm{~b}$. The E.D.50 for category 1 or more pneumoconiosis was found to be 72,000 exposure units [p.p. ml. (1-5 microns) $\times$ hours $\times$ months]. This means, for example, that if a group of men were to work a $7 \cdot 2$ hour shift in an average concentration of 100 p.p. ml. (1-5 microns) at the particular colliery for a period of 100 months they would have a $50 \%$ chance of developing category 1 or more pneumoconiosis. As the "measured" exposure is calculated in terms of the product of average concentration and time, this latter remark would apply equally well to a group of men working a $7 \cdot 2$ hour shift in an average concentration of 1,000 p.p. ml. for a period of 10 months, and similarly for any other combination of shift length, average concentration, and period of exposure of which the product comes to 72,000 . The E.D. 50 for category 2 or more pneumoconiosis was found to be 135,000 exposure units. It must be emphasized that no quantitative significance should be attached to these figures, as the accuracy of the estimates is poor because of the small amount of progression recorded.

The "fit" of the observations to the underlying assumptions on which the analysis is based was examined by comparing the observed and expected numbers of men showing the various types of response on the two surveys. The agreement was found to be good, and there was no evidence of any significant deviations. In this instance, of course, the amount of progression was small and little information could be obtained about whether or not the methods of analysis had dealt adequately with the "measured" component of exposure. However, the analysis provides a satisfactory example of the potential applications of the method.

This particular study was based on independent readings of the first and second survey films. By a suitable modification of the methods of calculation (Ashford et al., 1960) the same basic treatment may be applied to cover the situation in which the corresponding first and second survey films are classified together.

\section{Discussion}

The underlying object of each of the various procedures described above is to determine the direct relation between the radiological abnormality associated with simple pneumoconiosis and some single measure of past hazard. On the basis of this information it is possible to predict, with known accuracy, the abnormality value associated with any given exposure. By comparing the relationships associated with various alternative measures of exposure it will also be possible to determine which provides the most appropriate representation of the past hazard. In this way the main objective of the Pneumoconiosis Field Research, which is to determine safe levels of dustiness, will be achieved. In view of the uncertainties about the relation between radiological pneumoconiosis and disability and about the natural history of P.M.F. which exist at the present time, it would be inappropriate to attempt to lay down any specific criteria for safe levels at this stage. Various investigations which are currently in progress, both within the Pneumoconiosis Field Research and elsewhere, should help to resolve these uncertainties.

The methods of analysis which have been described are based on what is essentially a very simple representation of the relation between abnormality and exposure. The precise mechanisms involved in the development of simple pneumoconiosis may well be extremely complex, but it is assumed that an approach of this kind will provide an adequate description of the various processes involved. 


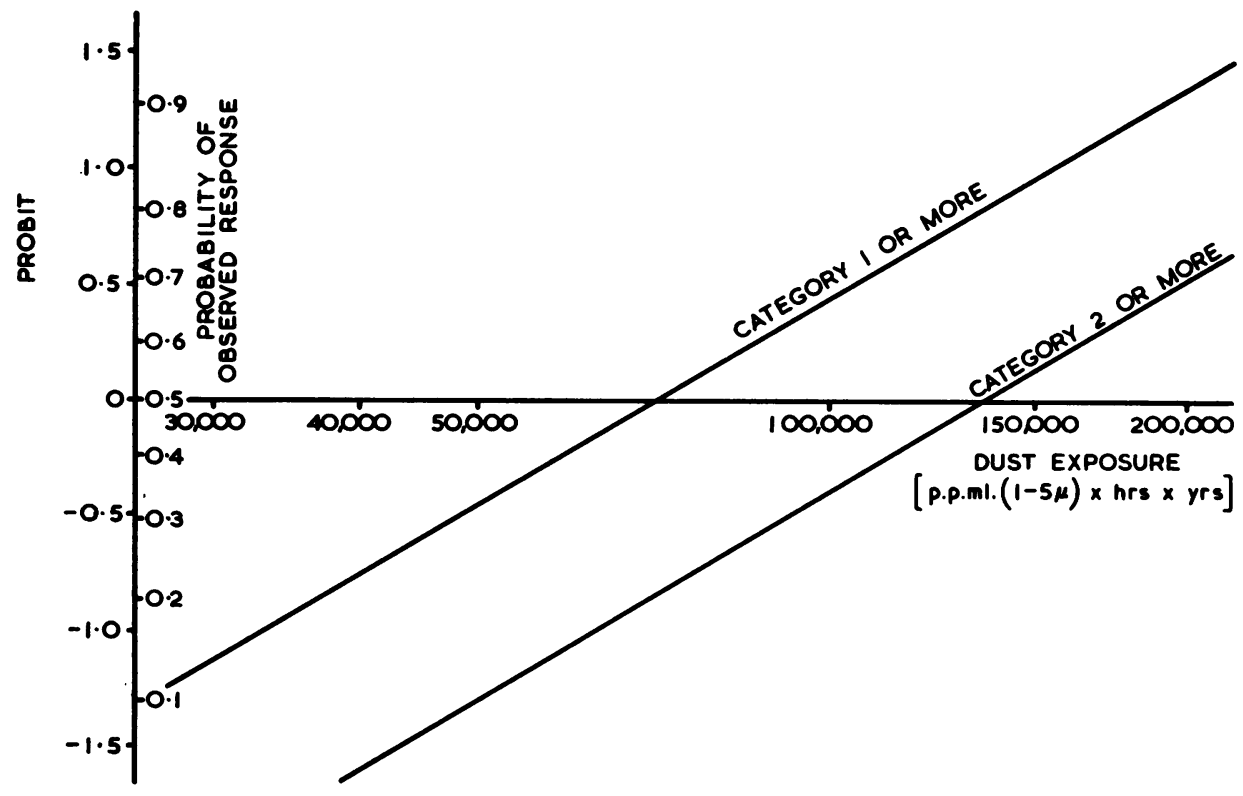

Fig. 7a

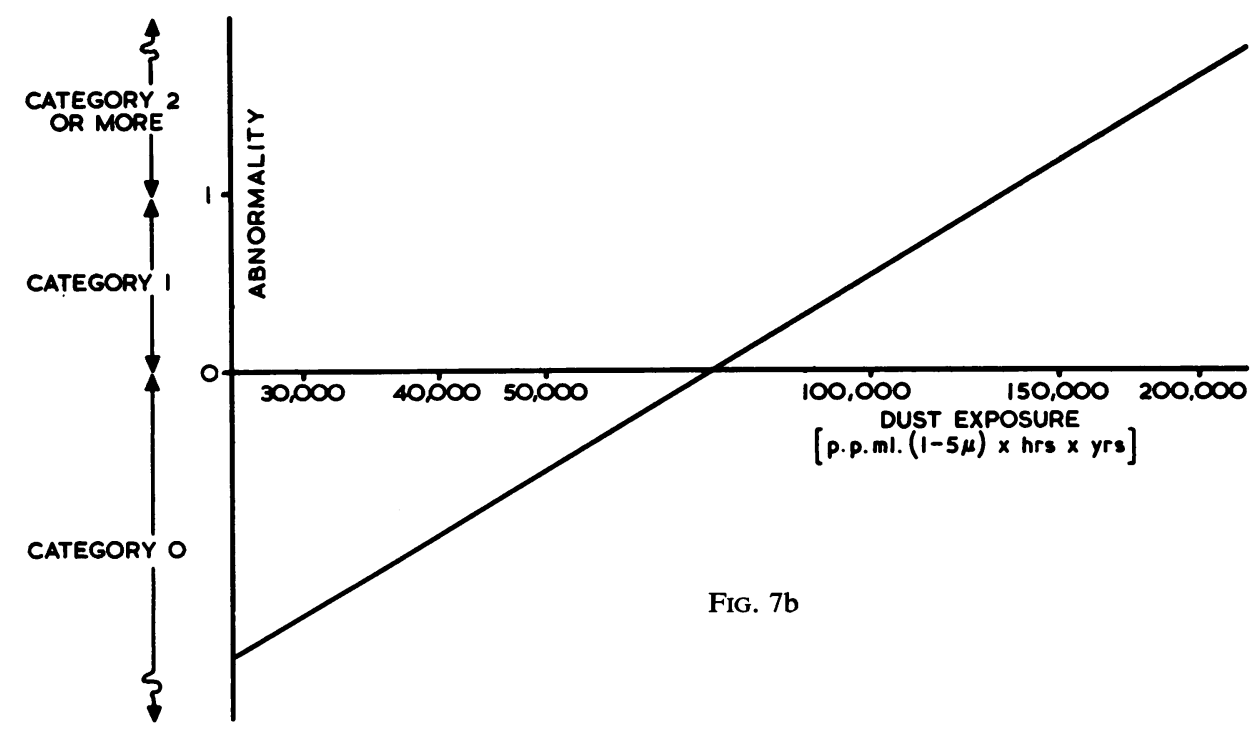

FIG. 7. Progression analysis based on the period spent in "dusty" occupations before the first survey and "measured" dust exposure between first and second surveys. (a) The quantal response; (b) The quantitative response.

Within the basic framework of the model it will, however, be possible to test a very wide variety of alternative hypotheses about the nature of the hazard, by varying the basis for calculating the "measured" component of exposure in the progres- sion analyses. In this respect it is perhaps unfortunate that no single hypothesis has yet emerged from all the basic research which has been undertaken into simple pneumoconiosis. It is desirable that any hypothesis which might be put forward as 
a result of these statistical analyses should be tested if possible by more fundamental studies before it is adopted as a basis for the control of dust levels.

In each separate analysis it is possible to check that the observed data are consistent with the various assumptions on which the treatment is based. A large number of analyses have been carried out in terms of the relation between the prevalence of pneumoconiosis at the time of the first medical surveys and the past working histories, when measured both as single- and multi-dimensional variables. The observed prevalences of pneumoconiosis have been compared with the corresponding values which would be expected if the model were exact. The frequency of occurrence of significant discrepancies between corresponding figures are very low, and certainly no greater than would have been expected as the result of random variations if the model were exactly true. It is therefore fair to conclude that the underlying assumptions are established, and that they can be applied with confidence in future analyses of the progression data. Nothing can be said about whether some alternative treatment would have led to an even better measure of agreement with the observations. It is, however, possible to assert that if this were the case, the alternative approach must necessarily agree very closely with the methods which have been described here. At this stage in the Research our experience of the progression analyses is limited, but the few results available are consistent with the assumed model. As the methods developed for the progression studies represent a natural extension of those adopted for the analysis of the data from the first round of medical surveys, it seems reasonable to presume that they will be useful in this rather more complicated application.

The use of the retrospective working history data forms an essential part of the methods developed for the progression studies. Although at first sight this approach might appear to be subject to some deficiencies, the analyses of the results of the first medical surveys have established that these working histories can, if expressed in a suitable form, provide a valid measure of past hazard. Since the period before the first medical surveys will account for the greater part of the total hazard of the majority of the men under examination for some years to come, there is an obvious need to take account of this contribution to the man's radiological abnormality.

The study of working populations to determine the relation between pneumoconiosis and past environment is subject to the criticism that they are survivor populations; any tendency for the presence or absence of pneumoconiosis to have an effect on whether a man joins or leaves the population will influence the observed results. However, the effect of simple pneumoconiosis, particularly in its early stages, on respiratory function is known to be small (Carpenter, Cochrane, Gilson, and Higgins, 1956; Rogan et al., 1961) and it is therefore probable that the selective withdrawal of men with minimal or even moderate simple pneumoconiosis will be negligible. This is confirmed by the studies of the dosage-response relationships based on the results of the first medical surveys. For these reasons it is thought that the methods of analysis described in this paper will not be affected appreciably by population selection. On the other hand, P.M.F. is known to be disabling and associated with a reduced expectation of life (Cochrane et al., 1956); it is therefore to be expected that studies of working populations will not provide a valid basis for the assessment of the natural history of complicated pneumoconiosis.

Finally, it must be pointed out that direct comparisons between dosage-response relationships based on two different populations of men involve the tacit assumption that both populations may be regarded as being selected at random from a single larger population. In other words, it must be assumed that there is no difference in the response pattern of the individual populations. Conclusive evidence in support of this assumption is difficult to obtain but on general grounds it seems likely to be justifiable and no evidence to the contrary has been found in any of the analyses.

The authors' thanks are due to Mr. D. Hicks, who directed this work, for his invaluable advice and encouragement, and to Dr. Stewart Rae for many helpful discussions. This paper is published by permission of the National Coal Board but the views expressed are not necessarily those of the Board. The work described is part of the Board's Pneumoconiosis Field Research.

\section{REFERENCES}

Ashford, J. R. (1958a). Biometrika, 45, 74

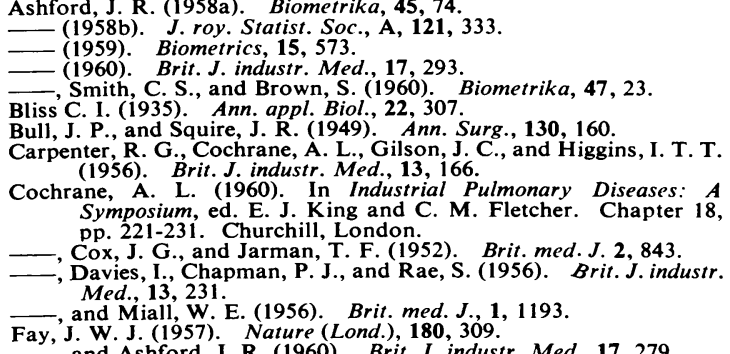


Fay, J. W. J., and Hicks, D. (1960). The Study of the Composition of Respirable Dust in the Pneumoconiosis Field Research. N.C.B. Report No. SC. 780/ER/125. (Unpublished.)

F-, and Rae, S. (1959). Ann. occup. Hyg., 1, 149. Fechner, G. T. (1861). Elemente der Psychophysik. Breikopf and Finney, D. J. (1952). Statistical Method in Biological Assay Griffin, London. Fletcher, C. M., and Oldham, P. D. (1949). Brit. J. industr. Med.,

Gough, J. (1947). Occup. Med., 4, 86.

Hart, P. D'Arcy, and Aslett, E. A. (1942). Spec. Rep. Ser. med. Res. Coun. (Lond.), No. 243.

Hicks, D., Fay, J. W. J., Ashford, J. R., and Rae, S. (1961). The Relation between Pneumoconiosis and Environmental Conditions:
An Analysis of the Results of the First Series of X-ray Surveys in the N.C.B's Pneumoconiosis Field Research. N.C.B. (Unpublished.)

International Labour Organization (1953). Third International Conference of Experts on Pneumoconiosis, Sydney, 1950. International Labour Office, Geneva.

Oldham, P. D., and Roach, S. A. (1952). Brit. J. industr. Med., 9, 112.

Patterson, H. S. (1939). Bull. Instn Min. Metall. (Lond.), No. 417. Rivers, D., Wise, M. E., King, E. J., and Nagelschmidt, G. (1960). Brit. J. industr. Med., 17, 87.

Roach, S. A. (1953). Ibid, 10, 220.

Rogan, J. M., Ashford, J. R., Chapman, P. J., Duffield, D. P., Fay, J. W. J., and Rae, S. (1961). Brit. med. J. 1, 1337

Stewart, A. (1948). 'Brit. J. industr. Med., 5, 120.

\section{THE APRIL (1961) ISSUE}

The April (1961) issue contains the following papers:-

“Sooty Manchester"' and the Social-Reform Novel 1845-1855. Arthur Pollard.

Chronic Respiratory Disease in a Random Sample of Men and Women in the Rhondda Fach in 1958. I. T. T. Higgins and A. L. Cochrane.

Carbon Pneumoconiosis. A. A. Miller and F. Ramsden.

Carbon Pneumoconiosis in a Synthetic Graphite Worker. W. B. Lister.

Byssinosis Among Cotton Workers in Belgium. E. Tuypens.

The Role of Bacterial Endotoxins in Occupational Diseases Caused by Inhaling Vegetable Dusts. B. Pernis,

E. C. Vigliani, C. Cavagna, and M. Finulli.

Histamine Liberation by Cotton Dust Extracts: Evidence Against its Causation by Bacterial Endotoxins. H. Antweiler.

Chronic Pulmonary Berylliosis in a Female Chemist. R. I. McCallum, I. Rannie, and C. Verity.

Observations on the Pathogenesis of Silicosis by Means of the Diffusion Chamber Technique. A. G. Heppleston, K. A. Ahlquist, and D. Williams.

The Effects of Vole Bacillus Vaccination of African Mine Workers in the Northern Rhodesian Copper Mines. R. Paul.

Cerebral Tumour in a Lead Worker. R. W. Portal.

Miscellanea :

Preliminary Communication: A Physiological Study of the Effects of Vibration on the Fingers. Ladislaus Magos.

Expired Air Resuscitation in a Contaminated Atmosphere. W. M. Dixon, A. Ward Gardner, and B. G. B. Lucas.

\section{Book Reviews}

A number of copies are still available and may be obtained from the Publishing Manager, British Medical Association, Tavistock Square, W.C.1, price 17s. $6 d$. 